\title{
Improving flexor tendon gliding by using the combination of carboxymethylcellulose-polyethylene oxide on murine model
}

\author{
ANDREEA MIOARA CORDUNEANU-LUCA ${ }^{1}$, SORIN AURELIAN PASCA ${ }^{2 *}$, \\ CAMELIA TAMAS ${ }^{3}$, DAN CRISTIAN MORARU ${ }^{3 *}$, BOGDAN CIUNTU $^{4}$, CRISTINA STANESCU $^{5}$, \\ IRINA HRENIUC-JEMNOSCHI ${ }^{3}$, ANGELA TECUCEANU ${ }^{3 *}$ and TEODOR STAMATE ${ }^{3}$
}

${ }^{1}$ Department of Plastic Surgery, 'Sf Ioan' Children's Hospital, 800487 Galati; ${ }^{2}$ Department of Veterinary Pathology, Faculty of Veterinary Medicine, 'Ion Ionescu de la Brad' University of Agricultural Sciences and Veterinary Medicine, 700490 Iasi; Departments of ${ }^{3}$ Plastic Surgery and ${ }^{4}$ General Surgery, Faculty of Medicine, 'Grigore T. Popa' University of Medicine and Pharmacy, 700115 Iasi; ${ }^{5}$ Department of Morphological and Functional Sciences, Faculty of Medicine and Pharmacy, 'Dunarea de Jos’ University, 800216 Galati, Romania

Received September 14, 2021; Accepted October 14, 2021

DOI: $10.3892 / \mathrm{etm} .2021 .11028$

\begin{abstract}
The current approach to flexor tendon injuries is complex and is no longer limited to suturing techniques. Strategies for improving hand function currently include rehabilitation protocols, appropriate suturing materials and techniques, changing the gliding surface by using lubricants and providing growth factors. One product, originally used in spinal surgery, has been shown to be effective in preventing postoperative adhesions. It is a combination of carboxymethylcellulose and polyethylene oxide-Dynavisc ${ }^{\circledR}$ (FzioMed, Inc.). The aim of the present study was to test the effect of Dynavisc ${ }^{\circledR}$ on acute injuries of the intrasynovial flexor tendons in the prevention of postoperative adhesions and the improvement of functional results. The study was performed on 20 Wistar rats distributed in two groups. The control group, represented by 10 rats, in which after the reconstruction of the flexor tendon, the peritendinous area was injected with saline solution and the study group, in which the peritendinous area was injected with a single administration of the lubricating gel, Dynavisc ${ }^{\circledR}$ (carboxymethylcellulose and polyethylene oxide). At 4 and
\end{abstract}

Correspondence to: Dr Bogdan Ciuntu, Department of General Surgery, Faculty of Medicine, 'Grigore T. Popa' University of Medicine and Pharmacy, 16 Universitatii Street, 700115 Iasi, Romania

E-mail: bogdan-mihnea.ciuntu@umfiasi.ro

Dr Camelia Tamas, Department of Plastic Surgery, Faculty of Medicine, 'Grigore T. Popa' University of Medicine and Pharmacy, 16 Universitatii Street, 700115 Iasi, Romania

E-mail: camelia.tamas@umfiasi.ro

${ }^{*}$ Contributed equally

Key words: flexor tendon, adhesions, carboxymethylcellulose and polyethylene oxide, lubricant, gliding
12 weeks, the rats were sacrificed and tissue biopsy consisted of tendon fragments and adjacent tissue. The evaluation of the results was performed by measuring the adhesion score and observing histological parameters. The presence of important adhesions was found in the control group compared with the group treated with Dynavisc ${ }^{\circledR}$, where a supple and smooth tendon, with significantly fewer adhesions were found. The differences between the two groups were significant, thus indicating the efficiency of the lubricant in preventing adhesions. This study supported the important role of Dynavisc ${ }^{\circledR}$ in the regeneration of the tendon and the peritendinous structures, by limiting aberrant fibrous proliferation in the regeneration process and helping to build a peritendinous space.

\section{Introduction}

The current approach to flexor tendon injuries is complex and is no longer limited to suturing techniques (1). A retrospective study performed in the United States over a 10 year period estimates that the incidence of acute flexor tendon injury is $\sim 33.2 / 100,000$ individuals per year (2). Tendons heal through a combination of two mechanisms: Intrinsic and extrinsic. Both follow similar stages to other tissues including inflammation lasting 48-72 h, fibroblast proliferation, collagen production for 3-4 weeks, and remodeling involving cross-linking and reduction of type III collagen with fiber reorientation (3). Extrinsic healing is characterized by rapid influx of fibroblasts from the peritendinous tissues, which favors the formation of adhesions, and intrinsic healing occurs through fibroblasts originating in the endotendon, without the formation of adhesions and which by early mobilization stimulates the synovial pumps, with a higher resistance on the suture line (4).

The most difficult tendon injury is zone II in which the adhesions of the flexor tendon radically influence the function of the hand. In zone II, located from the metacarpo-phalangeal joint to the base of the middle phalanx, is a narrow osteofibrous canal through which the two flexor tendons of the fingers pass. Tendon repair in this area predisposes to the formation of 
adhesions, as well as the appearance of diastasis (a weakening of the sutures with the removal of the tendon ends and not a complete rupture of them) or rupture. All this can compromise the postoperative functional result (5).

Strategies to improve hand function currently include postoperative rehabilitation protocols, appropriate suturing materials and techniques, changing the gliding surface by using lubricants and providing growth factors (4). Primary repair is indicated because secondary interventions involve difficult dissection of the vascular and nervous elements surrounded by fibrosis (6). When choosing a type of suture it must meet certain conditions, including: i) Be easy to perform; ii) reduce tendon manipulation; iii) minimize the amount of foreign material on the surface of the tendon; and iv) ensure sufficient strength for early mobilization (6). Regarding the manipulation of the biological environment, it has been studied on experimental models at the cellular, molecular and genetic levels, but they do not yet have large-scale clinical applicability, as described in a previous study by Evans (7). Reducing the abrasion resistance by modifying the exogenous surface can be achieved by applying lubricants, such as hyaluronic acid, lubricin and phospholipids (8-11).

Lubricin is a unique proteoglycan found in the fluid that lubricates the surface of intrasynovial tendons and it has been used in combination with hyaluronic acid to prevent synovial cell overgrowth. When used alone and not in combination with hyaluronic acid there were found to be no significant differences in adhesion prevention $(12,13)$.

The effects of hyaluronic acid on adhesions that form in the digital channel after reconstruction have been tested since the 1980s, when St Onge et al (14) applied a paste based on sodium hyaluronate to the repaired deep flexor tendon of fingers in monkeys. They compared the functional results with the control group to which saline was applied and found an improvement in total active motion in the studied group (14). The disadvantage regarding the application of hyaluronic acid on tendons is its rapid degradation by the body ( $\sim 7$ days), thus it requires repeated injection (15).

Another product, originally used in spinal surgery, has been shown to be effective in preventing postoperative adhesions. It is a combination of carboxymethylcellulose and polyethylene oxide-Dynavisc $^{\circledR}$ (FzioMed, Inc.). It is a transparent, synthetic, pure gel that does not contain animal proteins and does not interfere with tissue healing unlike other compounds of animal origin (16-18). This gel has proven its effectiveness especially in spinal surgery and neurolysis (surgical mobilization of the adherent nerve) (19-23) and has not been used in acute tendon injuries, opening up a research perspective in this regard. The aim of the present study was to test the effect of Dynavisc ${ }^{\circledR}$ on acute injuries of the intrasynovial flexor tendons in the prevention of postoperative adhesions and the improvement of functional results.

\section{Materials and methods}

Ethical approval. This study was approved by the Ethics Commission of 'Grigore T. Popa' University of Medicine and Pharmacy (Iasi, Romania; approval no. 20311/26.09.2019) in compliance with ethical and deontological rules for medical and research practice (24).
Experimental conditions. The experimental study was performed on 20 adult male Wistar laboratory rats (age, 6 months) provided from the Romanian National Institute of Research, weighing 300-350 g distributed in two groups. The housing conditions were as follows: i) Temperature average, $21^{\circ} \mathrm{C}$; ii) humidity, 46-65\%; and iii) light/dark cycle, $12 / 12 \mathrm{~h}$. Each animal had acces to food and water, and was kept in their own cage on the first postoperative day.

The control group, represented by 10 rats, in which after the reconstruction of the flexor tendon, the peritendinous area was injected with saline solution and the experimental group, in which the peritendinous area was injected with a single administration of lubricating gel, Dynavisc ${ }^{\circledR}$ (carboxymethylcellulose and polyethylene oxide). A total of five subjects from each group were sacrificed at 4 and 12 weeks when a biopsy was taken from the operated tendon. The evaluation of the results was performed by measuring the adhesion score (presence of peritendinous conjunctival hyperplasias marked with none, one, two or three + ) and by observing histological parameters.

Operative protocol. General anesthesia was performed preceded by intramuscular sedation. Xylazine (2\%) was used for sedation $(0.1 \mu \mathrm{g} / 100 \mathrm{~g}$ animal $)$, then ketamine ( $1 \mathrm{mg} / \mathrm{ml}-0.1 \mu \mathrm{g} / 100 \mathrm{~g}$ animal) was injected every $10-15 \mathrm{~min}$.

All substances were injected intramusculary on the thigh opposite the operated limb. In addition, for postoperative pain control, bupivacaine $(0.3 \mu \mathrm{g} / \mathrm{animal})$ was administered topically at a single dose to each experimental animal.

The animals were placed in a prone position and one of the hind limbs was disinfected with Betadine and then isolated with a sterile field. A longitudinal incision was made in the radius of finger three from the base to the tip of the finger with the exposure of the deep flexor tendon. Under magnification the flexor tendon and pulleys were sectioned, and then sutured with 9-0 thread using the modified Kessler technique (25) augmented with an epitendinous suture (Fig. 1). In the control group, the perilesional area and the digital osteo-fibrous canal were injected with $0.9 \%$ saline and in the experimental group, rats were injected with Dynavisc $^{\circledR}$ (0.05 $\mathrm{ml}$ each tendon). The lubricant was injected through a catheter. Then, the skin was sutured with 7-0 thread and a hydrofilm foil was applied to protect the wound.

Histology. At 4 and 12 weeks, the rats were sacrificed by an overdose of anesthetic. Halothane (1-5 ml/30-150 g) was used in a $50 \mathrm{ml}$ tube with some tissue to absorb easily and the head was placed inside the tube. The rats were placed in a closed chamber for $10 \mathrm{~min}$.

Tissue biopsy consisted of tendon fragments and adjacent tissue. Immediately after harvesting, the tissue fragments were fixed in $10 \%$ neutral buffered formalin solution for $48 \mathrm{~h}$ at room temperature, then processed by paraffin embedding method (Leica TP1020; Leica Microsystems GmbH) and sectioned longitudinally (microtome RM 2235; Leica Microsystems $\mathrm{GmbH}$ ), to a thickness of $5 \mu \mathrm{m}$. The sections were stained using Masson's stain at room temperature and examined with a photonic microscope (Leica DM 1000; Leica Microsystems $\mathrm{GmbH}$ ), using a digital histological camera (Leica $5 \mathrm{mpx}$, full HD; Leica Microsystems $\mathrm{GmbH}$ ) and LAS software, version 2016 (Leica Microsystems $\mathrm{GmbH}$ ) to capture images. Tendon 

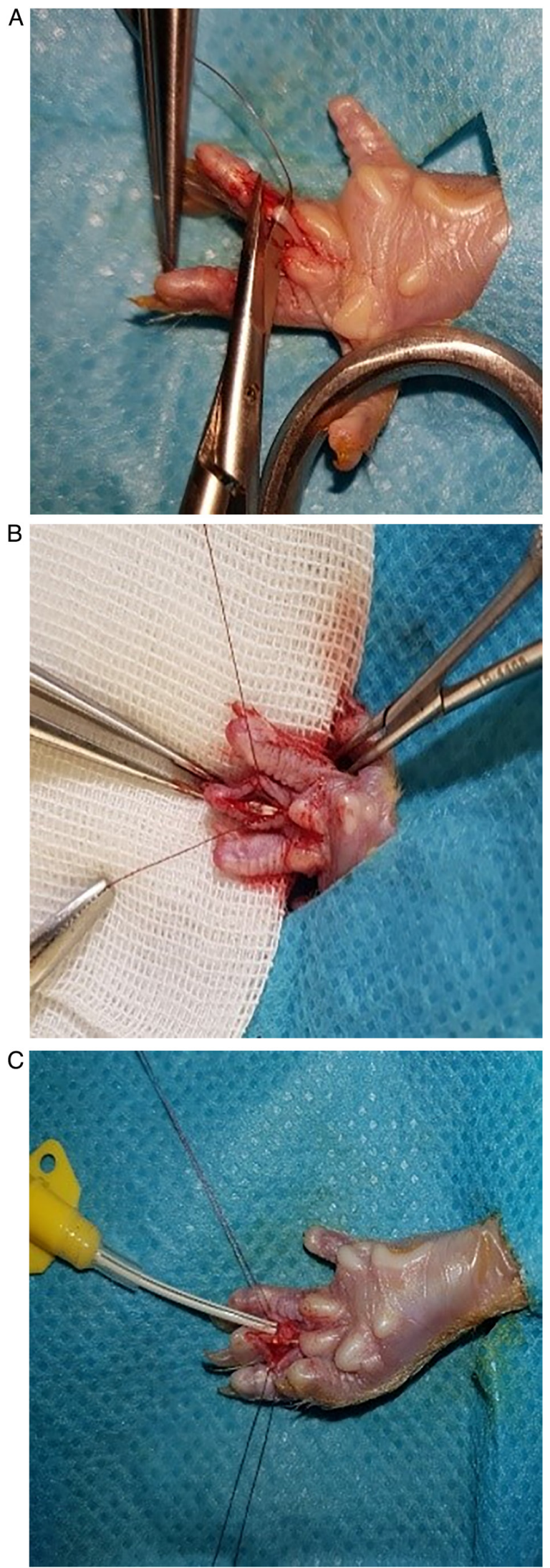

Figure 1. Intraoperative aspect. (A) Tendon cut and (B) suture with (C) application of lubricant.

healing was assessed by using the following indicators: i) The degree of proliferation of local tenocytes and fibroblasts; ii) the synthesis of collagen fibers in the perimeter of the tendon (actual regeneration of the tendon); iii) the presence of peritendinous conjunctival hyperplasia (fibrous adhesions); and iv) the presence of peritendinous space with the restoration of the external sheath of the tendon (epitenon) and the peritendinous synovial sheath.

Statistical analysis. For statistical analysis SPSS software version 19 (Statistical Packages for the Social Sciences), was used. The incidence of tendon rupture and the score of adhesions were calculated using the Fisher's exact test. The quantitative data are presented as the mean $\pm \mathrm{SD}$ (the presence of microscopic adhesions). $\mathrm{P}<0.05$ was considered to indicate a statistically significant difference.

\section{Results}

Clinical observation. In the control group a single rupture at 12 weeks and two tendons with diastasis at 4 weeks were recorded, while in the experimental group treated with Dynavisc $^{\circledR}$, three rats with diastasis (two in the 12 weeks group) and a single rupture in the group of 12 weeks were identified (Table I). The Fisher's exact test showed no significant differences between the two groups $(\mathrm{P}=0.871)$.

At the time of the biopsy, from an observational point of view, the presence of important adhesions were found in the control group, with infiltration of the tendon and adjacent tissues, which made it difficult to perform the biopsy, and a rough, opaque tendon with a low degree of mobility. In the experimental group, the presence of a supple and smooth tendon, with significantly less adhesions, was observed (Fig. 2).

Histology. From a histological point of view, in both groups, at 4 and 12 weeks, no notable differences were observed in terms of the actual recovery of the tendon. Notable fibrosis in the peritendinous tissue was noted in the control group compared with the experimental group. The presence of adhesions was notably lower in the experimental group, where a higher regeneration process was observed, characterized by much lower fibrous synechiae, which was absent in some animals, with the formation of a peritendinous fibrous tunnel, as a synovial sheath (Fig. 3).

Regarding frequency distribution of adhesions, the mean was 2.6 in the experimental group with a slight right-handed distribution compared with the control group where the mean was 2 with a right-tailed distribution (Fig. 4).

\section{Discussion}

Over the years, there have been numerous attempts to improve flexor tendon gliding and avoid complications by improving suturing techniques and recovery therapy (26). Despite these efforts, Strickland reported that between 30 and $40 \%$ heal through adhesions formation, which limits finger function (26). Wong et al (27) described the mechanism of adhesion formation on murine model and found that although intrasynovial tendons heal like a classic wound through inflamation, proliferation, synthesis and apoptosis, the greatest cellular activity is in the surrounding tissue where substantial scarring appears between tendon and the surrounding sheath (28).

If in the past only the surgical technique was given special importance, the current concepts also include the manipulation of the biological and biochemical environment to improve the 
Table I. Presence of rupture and diastasis in the two groups.

\begin{tabular}{lccccc}
\hline & \multicolumn{2}{c}{ Control group, $\mathrm{n}(\%)$} & & \multicolumn{2}{c}{ Experimental group, $\mathrm{n}(\%)$} \\
\cline { 2 - 3 } \cline { 5 - 5 } Complications & 4 weeks $(\mathrm{n}=5)$ & 12 weeks $(\mathrm{n}=5)$ & & 4 weeks $(\mathrm{n}=5)$ & 12 weeks $(\mathrm{n}=5)$ \\
\hline Diastasis & $2(20)$ & $0(0)$ & & $1(10)$ & $2(20)$ \\
Rupture & $0(0)$ & $1(10)$ & & $0(0)$ & $1(10)$ \\
\hline
\end{tabular}

Data were analyzed with Fisher's exact test, differences were not significant.
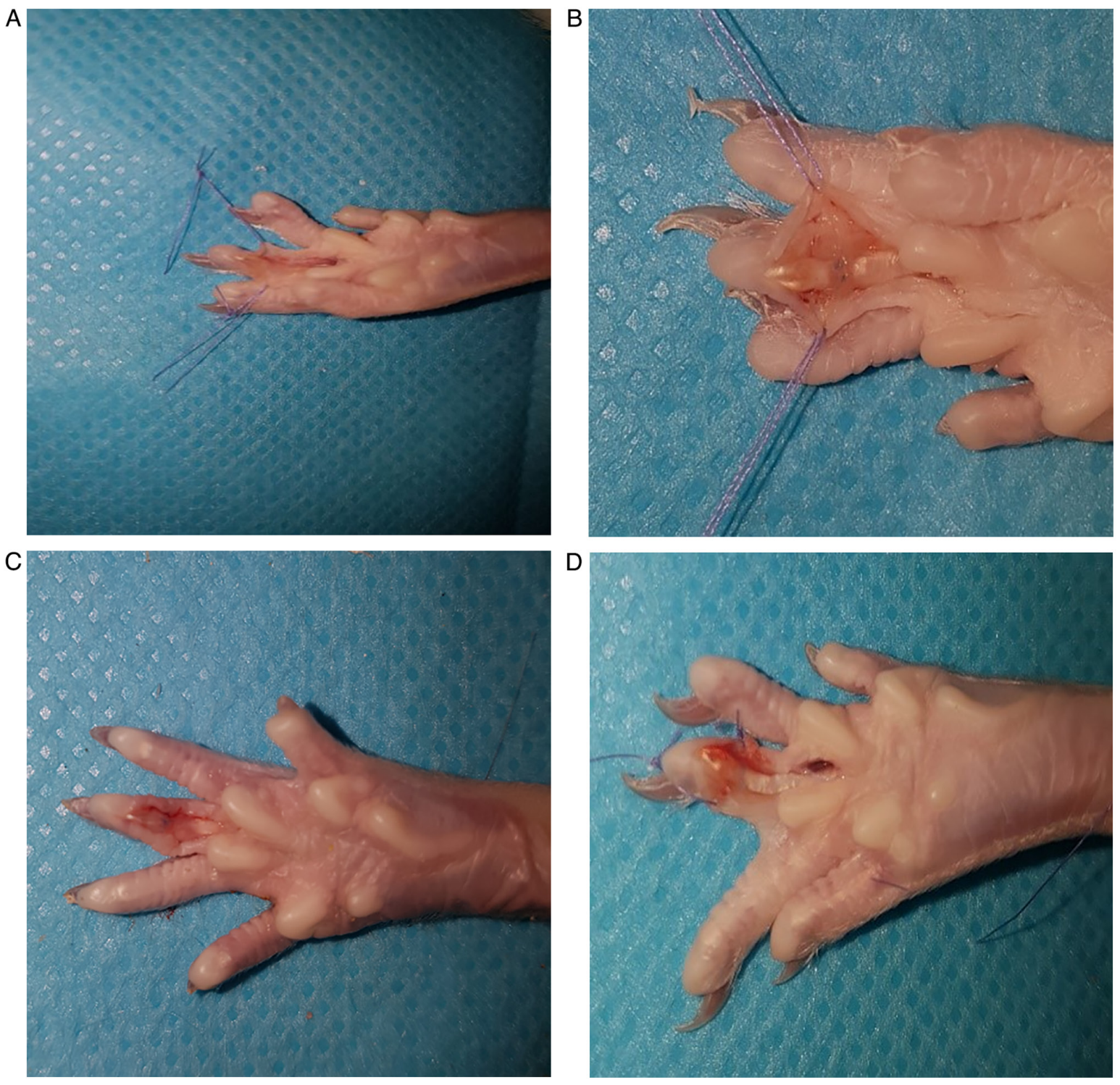

Figure 2. Observational difference between the two groups. In the control group, a rough surface with adhesions around the repaired tendon was observed at (A) 4 and (C) 12 weeks, and a smooth surface with no fibrosis in the experimental group was observed at (B) 4 and (D) 12 weeks.

response in intrinsic repair and diminish extrinsic healing as well as efforts to reduce frictional resistance of the tendon (28). Several adjuvants have been used to find the answer for rapid recovery after tendon surgery in experimental studies, but at present no human trials have been conducted (29). These adjuvants can be grouped in two categories: Drugs and barriers. The most popular drugs that have been invesigated include non-steroidal anti-inflammatory medications, 5-Fluorouracil, transforming growth factor $\beta$ inhibitors and lubricin. Among the barriers, the hyaluronic acid membrane, hydroxyapatite and bovine pericardia have been investigated the most (30-32).

Flexor tendons are intrasynovial tendons with a surface covered with a lubricating fluid that ensures the gliding in the narrow digital channel of the fingers, decreasing the frictional 

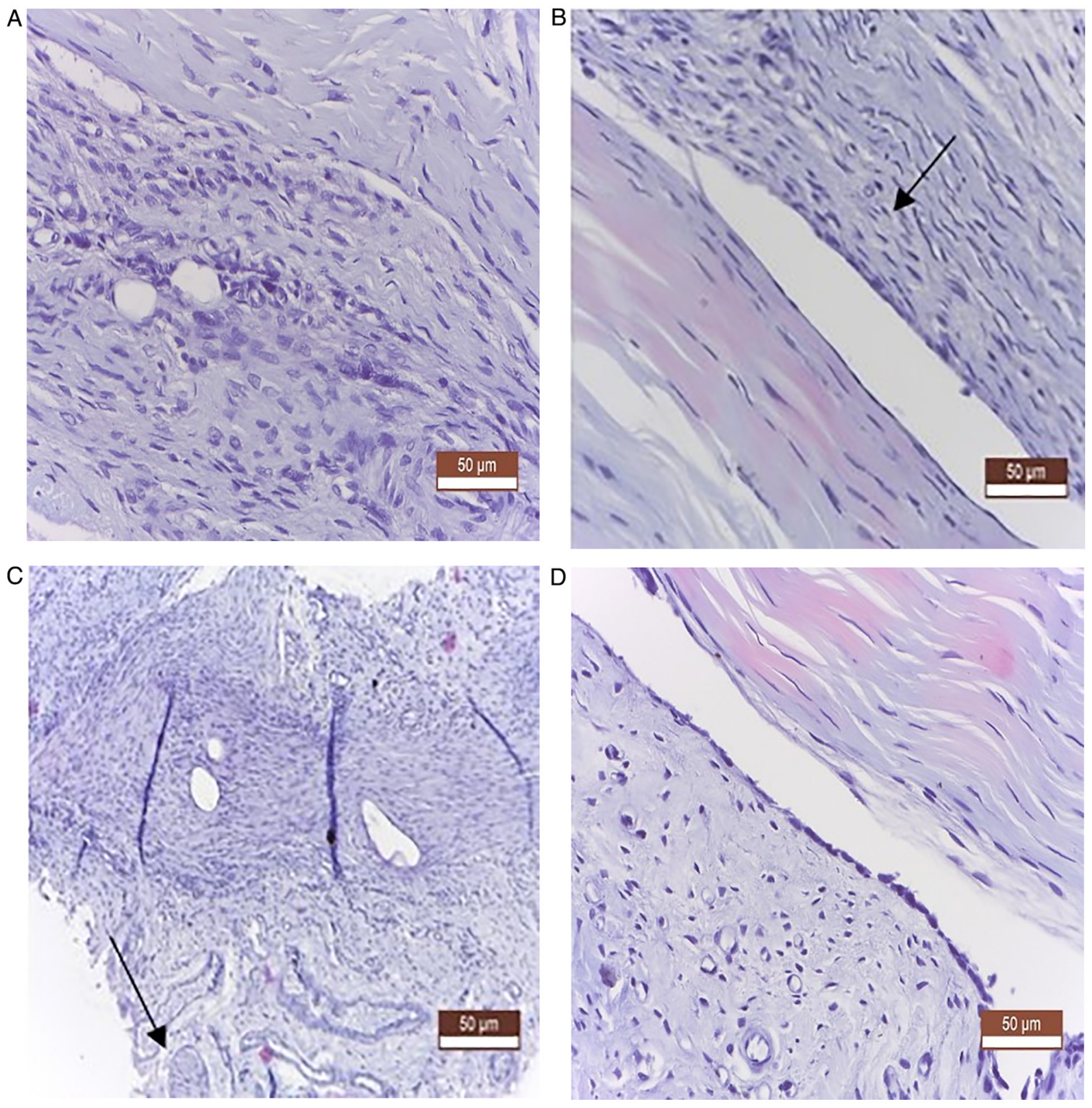

Figure 3. Histological appearance in the two groups following staining with Masson's trichrome stain. (A and C) Control group showed tenocyte proliferation and collagenization (vicious fibrosis that led to the fusion of the tendon with adjacent structures-conjunctival adhesions) and sequestration in the peritendinous connective mass of nerve fibers (arrow). (B and D) Experimental group showed fibroblast hyperplasia and epitenon fusion with peritendinous tissue (arrow), well-formed peritendinous space, with the role of a restored synovial sheath.

resistance between it and its sheath (33). Hagberg et al (34) studied the composition of fluid in the synovial sheath and found that it has a composition similar to the synovial fluid of the joints, being rich in hyaluronic acid with a role in lubrication and nutrition.

Hyaluronic acid has been shown to not influence tendon healing and not increase the risk of diastasis or rupture. This was proved by Özgenel and Etöz in a clinical study on 22 patients and they concluded that repetitive injections of hyaluronic acid can improve clinical outcomes when compared with a single dose administration (35).

The combination of carboxymethylcellulose-polyethylene oxide (Dynavisc ${ }^{\circledR}$ ) has been shown to be safe and effective in spinal and abdominal surgery and does not interfere with tissue healing. This has been confirmed by both experimental and clinical studies that have demonstrated the role of the barrier $(36,37)$.
The product is not metabolized by the body as in the case of hyaluronic acid so no reinjection is required.

In the present study, the activation and proliferation of tenocytes and resident fibroblasts at both ends of the tendon and the synthesis of new connective tissue between its ends were noted in both groups, so Dynavisc ${ }^{\circledR}$ did not inhibit tendon healing. In the control group, conjunctival hyperplasia with a repairing role was more intense and at the same time aberrant, occurring on the surface of the epitenon, invading the adjacent anatomical structures, surrounding the peripheral nerves. The synovial sheath disappeared by welding its two sheets and the disappearance of the mesothelium (internal visceral and parietal epithelium). There was also a large epithelioid-inflammatory reaction around the sutures in the thickness of the tendon. In the experimental group treated with Dynavisc ${ }^{\circledR}$, a 

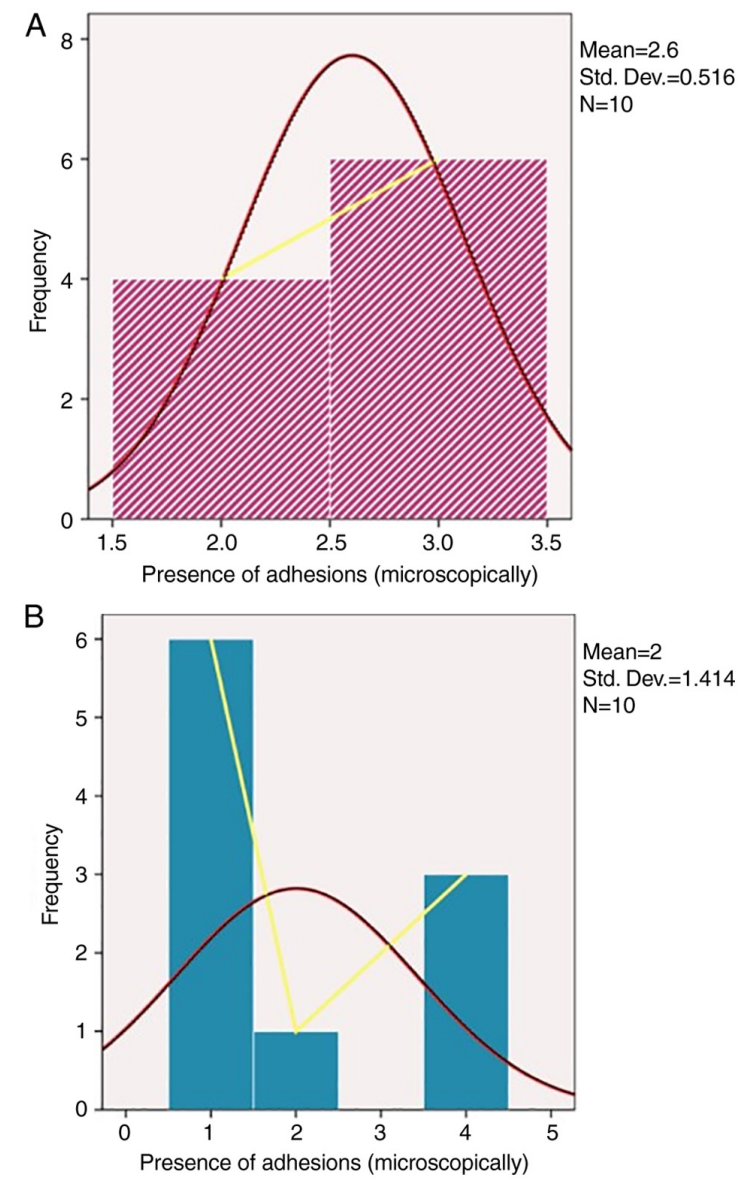

Figure 4. Frequency distribution for presence of adhesions. (A) Experimental and (B) control groups.

superior regeneration process was observed, characterized by much smaller fibrous synechiae, which was absent in some animals, with the constitution of a peritendinous fibrous tunnel with the role of synovial sheath. There were also areas of fibrillar neogenesis from the epitenon, but smaller, in terms of fibrous spicules or thin fibrous clamps.

It has been suggested that some products used to prevent adhesions may interfere with scar reshaping and complications. In the current study, the statistical analysis showed no significant differences between the two groups, which indicated that Dynavisc ${ }^{\circledR}$ did not influence the occurrence of complications during surgery, in terms of tendon healing.

From a histological point of view, in the present study case, in both groups, both at 4 and 12 weeks, no notable differences were noticed in terms of the actual recovery of the tendon. However, significant fibrosis in the peritendinous tissue was observed in the control group, compared with the experimental group. The major differences were found at 12 weeks where, in the control group, aberrant peritendinous conjunctival hyperplasias were identified, with a fibrous block appearance, with welding the body of the tendon at the adjacent tissues, also tightening regional nerve fibers (peripheral nerves). The existence of a peritendinous space with the role of synovial sheath was not noticed. In the experimental group at 12 weeks, these adhesions were notably reduced compared with the control group, so the lubricant did not delay the remodeling of the tendon scar.
The results of the current study suggested that a lack of lubricant administration leads to a high presence of macroscopic and microscopic adhesions. To the best of our knowledge, there are no other studies evaluating the effect of Dynavisc $^{\circledR}$ on the prevention of flexor tendon adhesions after primary repair, which is one of the strengths of the present study. In the future, this product could be tested in clinical practice, opening novel perspectives for improving tendon gliding after flexor tendon repair. The study has several limitations and in the future it could be improved by quantitative assays, particularly to evaluate the degree of proliferation of tenocytes and fibroblasts, and collagen synthesis. Also, this study did not include any biomechanical tests to evaluate the friction forces necessary to mobilize the tendon inside the digital canal, a parameter that can predict the limb function.

To conclude, biological approaches to prevent adhesion formation represent the future of acute flexor tendon surgery to obtain improved functional results. This study supported the important role of biological lubricant in the regeneration, not only of the tendon, but of the peritendinous structures, by limiting aberrant fibrous proliferation in the regeneration process and helping to build a peritendinous space, which will later form a true synovial sheath. In conclusion Dynavisc ${ }^{\circledR}$ can be used safely in acute flexor tendon injuries.

\section{Acknowledgements}

Not applicable.

\section{Funding}

No funding was received.

\section{Availability of data and materials}

All data generated or analyzed during this study are included in this published article.

\section{Authors' contributions}

AMCL, CS and DCM performed the experiments in the present study. AMCL, SAP, CT, TS and BC performed data analysis. AMCL, SAP, DCM, BC, IHJ, AT and TS wrote the manuscript. CS, DCM, IHJ and AT made substantial contributions to conception and design, acquisition, analysis and interpretation of data. AMCL, CS and DCM confirm the authenticity of all the raw data. All authors have read and approved the final manuscript.

\section{Ethics approval and consent to participate}

The study was approved by the Ethics Commission of 'Grigore T. Popa' University of Medicine and Pharmacy (Iasi, Romania; approval no. 20311/26.09.2019) in compliance with ethical and deontological rules for medical and research practice.

\section{Patient consent for publication}

Not applicable. 


\section{Competing interests}

The authors declare that they have no competing interests.

\section{References}

1. Chen $\mathrm{CH}$, Chen SH, Shalumon KT and Chen JP: Prevention of peritendinous adhesions with electrospun polyethylene glycol/polycaprolactone nanofibrous membranes. Colloids Surf B Biointerfaces 133: 221-230, 2015.

2. de Jong JP, Nguyen JT, Sonnema AJ, Nguyen EC, Amadio PC and Moran SL: The incidence of acute traumatic tendon injuries in the hand and wrist: A 10-year population-based study. Clin Orthop Surg 6: 196-202, 2014.

3. Wu YF and Tang JB: Tendon healing, edema, and resistance to flexor tendon gliding: Clinical implications. Hand Clin 29: $167-178,2013$

4. Sammer DM and Chung KC: Advances in the healing of flexor tendon injuries. Wound Repair Regen 22 (Suppl 1): S25-S29, 2014.

5. Loiselle AE, Kelly M and Hammert WC: Biological augmentation of flexor tendon repair: A challenging cellular landscape. J Hand Surg Am 41: 144-149, 2016.

6. Giesen T, Calcagni M and Elliot D: Primary flexor tendon repair with early active motion: Experience in Europe. Hand Clin 33: 465-472, 2017.

7. Evans RB: Managing the injured tendon: Current concepts. J Hand Ther 25: 173-190, 2012

8. Moro-oka T, Miura H, Mawatari T, Kawano T, Nakanishi Y, Higaki $\mathrm{H}$ and Iwamoto $\mathrm{Y}$ : Mixture of hyaluronic acid and phospholipid prevents adhesion formation on the injured flexor tendon in rabbits. J Orthop Res 18: 835-840, 2000.

9. Sun Y, Chen MY, Zhao C, An KN and Amadio PC: The effect of hyaluronidase, phospholipase, lipid solvent and trypsin on the lubrication of canine flexor digitorum profundus tendon. J Orthop Res 26: 1225-1229, 2008.

10. Taguchi M, Zhao C, Sun YL, Jay GD, An KN and Amadio PC: The effect of surface treatment using hyaluronic acid and lubricin in the gliding resistance of human extrasynovial tendons in vitro. J Hand Surg Am 34: 1276-1281, 2009.

11. Oryan A, Moshiri A and Meimandiparizi AH: Effects of sodium-hyaluronate and glucosamine-chondroitin sulfate on remodeling stage of tenotomized superficial digital flexor tendon in rabbits: A clinical, histopathological, ultrastructural, and biomechanical study. Connect Tissue Res 52: 329-339, 2011.

12. Rhee DK, Marcelino J, Baker M, Gong Y, Smits P, Lefebre V, Jay GD, Stewart M, Wang H, Warman ML and Carpten JD: The secreted glycoprotein lubricin protects cartilage surfaces and inhibits synovial cell overgrowth. J Clin Invest 115: 622-631, 2005.

13. Amadio PC: Gliding resistance and modifications of gliding surface of tendon: Clinical perspectives. Hand Clin 29: 159-166, 2013.

14. St Onge R, Weiss C, Denlinger JL and Balazs EA: A preliminary assessment of Na-hyaluronate injection into 'no man's land' for primary flexor tendon repair. Clin Orthop Relat Res 146 $269-275,1980$.

15. Amiel D, Ishizue K, Billings E Jr, Wiig M, Vande Berg J, Akeson WH and Gelberman MD: Hyaluronan in flexor tendon repair. J Hand Surg Am 14: 837-843, 1989.

16. Hayashi M, Zhao C, Thoreson AR, Chikenji T, Jay GD, An KN and Amadio PC: The effect of lubricin on the gliding resistance of mouse intrasynovial tendon. PLoS One 8: e83836, 2013.

17. Zhao C, Hashimoto T, Kirk RL, Thoreson AR, Jay GD, Moran SL, An KN and Amadio PC: Resurfacing with chemically modified hyaluronic acid and lubricin for flexor tendon reconstruction. J Orthop Res 31: 969-975, 2013.

18. Zhao C, Sun YL, Kirk RL, Thoreson AR, Jay GD, Moran SL, An KN and Amadio PC: Effects of a lubricin-containing compound on the results of flexor tendon repair in a canine model in vivo. J Bone Joint Surg Am 92: 1453-1461, 2010.

19. Kitano T, Zerwekh JE, Edwards ML, Usui Y and Allen MD: Viscous carboxymethylcellulose in the prevention of epidural scar formation. Spine (Phila Pa 1976) 16: 820-823, 1991.

20. Fransen P: Safety of carboxymethylcellulose/polyethylene oxide for the prevention of adhesions in lumbar disc herniation-consecutive case series review. Ann Surg Innov Res 2: 2, 2008.
21. Rodgers KE, Robertson JT, Espinoza T, Oppelt W, Cortese S, diZerega GS and Berg RA: Reduction of epidural fibrosis in lumbar surgery with Oxiplex adhesion barriers of carboxymethylcellulose and polyethylene oxide. Spine J 3: 277-284, 2003.

22. Gill C, Klufmoeller F and Noack W: Experience with Oxiplex/SP gel for the prevention of post-surgical adhesions in decompressive spine surgery. Paper presented at North American Spine Society 18th Annual Meeting. October 21-25, San Diego, CA, 2003.

23. Tos P, Crosio A, Pellegatta I, Valdatta L, Pascal D, Geuna S and Cherubino M: Efficacy of anti-adhesion gel of carboxymethylcellulose with polyethylene oxide on peripheral nerve: Experimental results on a mouse model. Musce Nerve 53: 304-309, 2016.

24. Balls M, Goldberg A, Fentem J, Broadhead CL, Burch RL, Festing MF, Frazier JM, Hendriksen CF, Jennings M, van der Kamp MD, et al: The three Rs: The way forward: The report and recommendations of ECVAM Workshop 11. Altern Lab Anim 23: 838-866, 1995.

25. Savage R: The search for the ideal tendon repair in zone 2: Strand number, anchor points and suture thickness. J Hand Surg Eur Vol 39: 20-29, 2014.

26. Strickland JW: The scientific basis for advances in flexor tendon surgery. J Hand Ther 18: 94-111, 2005.

27. Wong JKF, Lui YH, Kapacee Z, Kadler KE, Fergusos MWJ and McGrouther DA: The cellular biology of flexor tendon adhesion formation: An old problem in a new paradigm. Am J Pathol 175: 1938-1951, 2009.

28. Ozgenel GY: The effects of a combination of hyaluronic and amniotic membrane on the formation of peritendinous adhesions after flexor tendon surgery in chickens. J Bone Joint Surg Br 86: 301-307, 2004.

29. Khanna A, Friel M, Gougoulias N, Longo UG and Maffulli N: Prevention of adhesions in surgery of the flexor tendons of the hand: What is the evidence? Br Med Bull 90: 85-109, 2009.

30. Loiacono C, Palermi S, Massa B, Belviso I, Romano V, Gregorio AD, Sirico F and Sacco AM: Tendinopathy: Pathophysiology, therapeutic options, and role of nutraceutics. A narrative literature review. Medicina (Kaunas) 55: 447, 2019.

31. Megerle K, Woon C, Kraus A, Raghavan S, Pham H and Chang J: Flexor tendon sheath engineering using decellularized porcine pericardium. Plast Reconstr Surg 138: 630e-641e, 2016.

32. Linderman SW, Gelberman RH, Thomopoulos S and Shen H: Cell and biologic-based treatment of flexor tendon injuries. Oper Tech Orthop 26: 206-215, 2016.

33. Ji X, Reisdorf RL, Thoreson AR, Berglund LR, Moran SL, Jay JD, An KN, Amadio PC and Zhao C: Surface modification with chemically modified synovial fluid for flexor tendon reconstruction in a canine model in vivo. J Bone Joint Surg Am 97: 972-978, 2015.

34. Hagberg L, Heinegård D and Ohlsson K: The contents of macromolecule solutes in flexor tendon sheath fluid and their relation to synovial fluid. A quantitative analysis. J Hand Surg Br 17: 167-171, 1992.

35. Özgenel GY and Etöz A: Effects of repetitive injections of hyaluronic acid on peritendinous adhesions after flexor tendon repair: A preliminary randomized, placebo-controlled clinical trial. Ulus Travma Acil Cerrahi Derg 18: 11-17, 2012.

36. diZerega GS, Cortese S, Rodgers KE, Block KM, Falcone SJ, Juarez TG and Berg R: A modern biomaterial for adhesion prevention. J Biomed Mater Res B Appl Biomater 81: 239-350, 2007.

37. Lei W, Ehmsen RJ, Chiaccherini RP, Krelle JL and diZerega GS Reduction of leg pain by Oxiplex gel after lumbar discectomy in patients with predominant leg pain and elevated levels of lower back pain: A prospective, randomized, blinded, multicenter clinical study. J Spine Disord Tech 28: 301-307, 2015. International (CC BY-NC-ND 4.0) License. 